\title{
Stat proteins as intracellular regulators of resistance to myocardial injury in the context of cardiac remodeling and targeting for therapy
}

\author{
Andrzej Wincewicz ${ }^{1, D-F}$, Stanisław Sulkowski2, E,F \\ 1 Specialist Medical Practice-Pathologist, Non-Public Health Care Unit, Department of Pathology, Kielce, Poland \\ ${ }^{2}$ Department of General Pathomorphology, Medical University of Bialystok, Poland \\ A - research concept and design; $B$ - collection and/or assembly of data; $C$ - data analysis and interpretation; \\ $D$ - writing the article; $E$ - critical revision of the article; $F$ - final approval of article
}

Address for correspondence

Andrzej Wincewicz

E-mail:andwinc@gmail.com

Funding sources

None declared

Conflict of interest

None declared

Received on February 04, 2016

Revised on March 23, 2016

Accepted on April 15, 2016

\begin{abstract}
The roles of STAT (signal transducers and activators of transcription) proteins are widely discussed in relation to other agents like IFN- $y$ that are involved in cardiovascular diseases. STAT3 protects cardiomyocytes during endotoxic shock and ischemia and prolongs survival of these cells by activation of antiapoptotic genes like Bcl-2 and c-Fos. Moreover, LL-6 dependent expression of STAT3 is probably responsible for hypertrophy of cardiomyocytes. On the contrary, STAT1 mediates cell death by induction of caspase-1. STAT6 probably enhances cellular damage in myocardial infraction, which is significantly reduced in mice with the knockout STAT6 gene. Considering these facts, we attempted to review in this paper the role of STAT proteins in myocardial remodeling, highlighting STAT3 as a potent mediator of cardioprotection. Our review also aims to acquaint a broad audience of internal medicine practitioners with the STAT3-related molecular mechanisms that underlie the therapeutic properties of such widely administered drugs as angiotensin II type 1 (AT1) receptor antagonists and HMG-CoA reductase inhibitors, such as losartan and lovastatin.
\end{abstract}

Key words: apoptosis, signal transduction, cardiac remodeling, therapeutic implications

DOI

10.17219/acem/62693

Copyright

Copyright by Author(s)

This is an article distributed under the terms of the

Creative Commons Attribution Non-Commercial License

(http://creativecommons.org/licenses/by-nc-nd/4.0/) 
STAT (signal transducers and activators of transcription) proteins transduce signals from the cell membrane to the nucleus. They also induce gene transcription. There are 7 members of the STAT protein family: STAT1, STAT2, STAT3, STAT4, STAT5a, STAT5b and STAT6. We can distinguish a few functional sites of the STAT protein. The first one is a SH2 ( $\mathrm{Src}$ Homology 2) domain that binds a membrane receptor (usually gp-130 in the case of STAT3). The transactivation domain interacts with nuclear DNA. A C-terminal end of the STAT protein contains tyrosine and serine residues. ${ }^{1}$ Phosphorylation of the tyrosine residue activates STAT proteins which translocate from cytoplasm to nucleus. A serine residue acquires phosphate indirectly by kinases MAP (mitogen activated kinases), e.g. ERK1 (Extracellular Regulated Kinase), ERK2, p38, JNK (c-Jun N-terminal/stress-activated protein kinase) or H7-SK (H7-Sensitive Kinase), but STAT2 does not contain the serine residue. ${ }^{2,3}$ Phosphoserine contributes to the recruitment of transcription cofactors, for instance coactivator CBP. ${ }^{4}$ Janus kinases (JAK) are necessary for the type II cytokine receptors to activate STAT. However, receptors of tyrosine kinase activity (e.g. EGFR - Epidermal Growth Factor Receptor and PDGFR - Platelet-Derived Growth Factor Receptor) can stimulate STAT without the involvement of any intermediate messengers. ${ }^{1}$ In that case, STATs join to form dimmers and enter the nucleus, where they regulate the transcription of certain genes. As a result, they control the biosynthesis of their surface antigens, enzymes and other proteins. STAT proteins are involved in differentiation, proliferation, apoptosis and angiogenesis. ${ }^{1,5}$

\section{The antiapoptotic properties of STAT}

Cultured murine cardiac myocytes express the mRNA of c-Fos and ANF. These factors contribute to myocardial hypertrophy only if a function of STAT3 that is mediated by gp130 after stimulation by IL- 6 is not impaired by, for example, an adenoviral introduction of mutatedtype STAT3 cDNA into cardiomyocytes. ${ }^{6}$ In addition, dominant-negative STAT3 limited the expression of VEGF mRNA in the cultured cardiomyocytes of 1-2-dayold Wister rat lines after the stimulation of leukemia inhibitory factor (LIF) and also inhibited the expression of VEGF which was not induced by LIF in mutant transgenic mice with the STAT3 knockout gene. Therefore STAT3 is thought to take part in the angiogenic processes of cardiac vasculature. ${ }^{7}$ Transgenic (TG) mice with constitutively active STAT3 (caSTAT3) had more developed capillary vasculature of the heart and express VE-cadherin on endothelial cells very intensively. ${ }^{8}$ Moreover, development of the vascular network is remarkably impaired in mice without the STAT3 gene, which explains why these animals were more sensitive to ischemic damage and suffered from myocardial infractions to a greater extent than mice with functional molecules of STAT3. ${ }^{9}$ In rat neonatal cardiomyocytes, constitutively active STAT3 causes cytoprotective effects in case of hypoxia and following reoxygenation that, in vivo, result from ischemia and reperfusion. ${ }^{10}$ STAT3 upregulates expression of manganese superoxide dismutase (MnSOD), which is completely suppressed in cardiomyocytes by a transfection of the dominant negative STAT3. Less creatine phosphokinase escapes from cardiomyocytes after exposure to reactive oxygen species only if caSTAT3 was introduced in these cells. Thus, damage of cardiac myocytes seems to be limited by the detoxicative properties of STAT3 that are aimed at ROS. ${ }^{10}$ Overexpressed cardiac-specific STAT3 (STAT3-TG) upregulates production of atrial natriuretic factor (ANF), $\beta$-myosin heavy chain (MHC), and cardiotrophin (CT)-1 in rat cardiomyocytes. ${ }^{11}$ A cell injury that is generated by the antitumor agent doxorubicin is effectively attenuated by STAT3-TG, whose deficiency limits the survival of mice that were treated with this drug. ${ }^{12}$ In human end-stage dilated cardiomyopathy (DCM), there was a lower activity of JAK2 and a diminished immunoreactivity of STAT3 in cardiomyocytes, which indicates a possible disturbance of the gp-130 mediated JAK2/STAT3 pathway. ${ }^{13}$ A STAT3deficient murine myocardium was far more sensitive to the inflammatory damage caused by lipopolysaccharide in comparison with hearts with a normal expression of the gene. The knockout of STAT3 resulted in an increase of fibrosis and cardiac myocytes' apoptosis as a consequence of the upregulated production of TNF- $\alpha$ by cardiomyocytes. ${ }^{14}$ A delayed tyrosine phosphorylation of STAT3 is mediated indirectly by angiotensin II (AngII) via induction of IL-6, CT-1 and LIF mRNA expression. Subsequently, these factors are released by cardiomyocytes and can affect them in an autocrine or paracrine way, which results in activation of STAT3. ${ }^{15}$ Angiotensin II stimulates STAT1 and STAT2 by recruitment of JAK2 and Tyk2 in half an hour, and later, after $2 \mathrm{~h}$, it independently contributes to the phosphorylation of STAT3 ${ }^{16}$ Endothelin-1 interferes in the LIF-induced JAK/STAT pathway. However, despite the abrogation of STAT3, endothelin-1 causes hypertrophy of cardiac myocytes in an independent manner. ${ }^{17,18}$ In one study, it was found that STAT3 switched on survival gene Bcl-2 and inhibited expression of the counteracting BAX gene, which reduced the amount of cardiomyocytes that were undergoing cell death in circumstances of hypoxia and subsequent reoxygenation. ${ }^{16}$

\section{The proapoptotic function of STAT proteins}

STAT1 mediates the cell death of cardiomyocytes via induction of the caspase- 1 gene in response to IFN- $\gamma$ and in response to ischemia and reperfusion. ${ }^{19}$ Serine-727 phosphorylation of STAT1 contributes to expression of the Fas ligand and Fas receptor, whose signals determine 
cell death, and are transmitted after coupling this receptor with an activator of caspases - the FADD protein that is also stimulated by TNF- $\alpha .^{20}$ The antiapoptotic effects were attributed to the LIF-induced expression of Bcl-xL with very questionable signal mediation by STAT1 through gp130. ${ }^{21}$ Later studies clarified that gp-130 is associated with STAT3 rather than STAT1 and that cardiothropin-1 (CT-1) is a cytokine that antagonizes the gp-130 receptor and stimulates STAT3 and c-Fos-dependent survival of cardiomyocytes. ${ }^{7,8,11}$ As opposed to the cardioprotective involvement of STAT3 and probably STAT5a in ischemic preconditioning, the STAT6 gene is also activated in ischemia and reperfusion events. ${ }^{17,18,24}$ Namely, when the STAT6 gene was completely knocked out of mice, it resulted in a limitation of myocardial infraction. ${ }^{24}$ So STAT6 seems to have reverse properties to STAT3 and STAT5, which regulate the survival of cardiomyocytes.

Fig. 1. Impact of STAT1 and STAT3 on cardiomyocytes

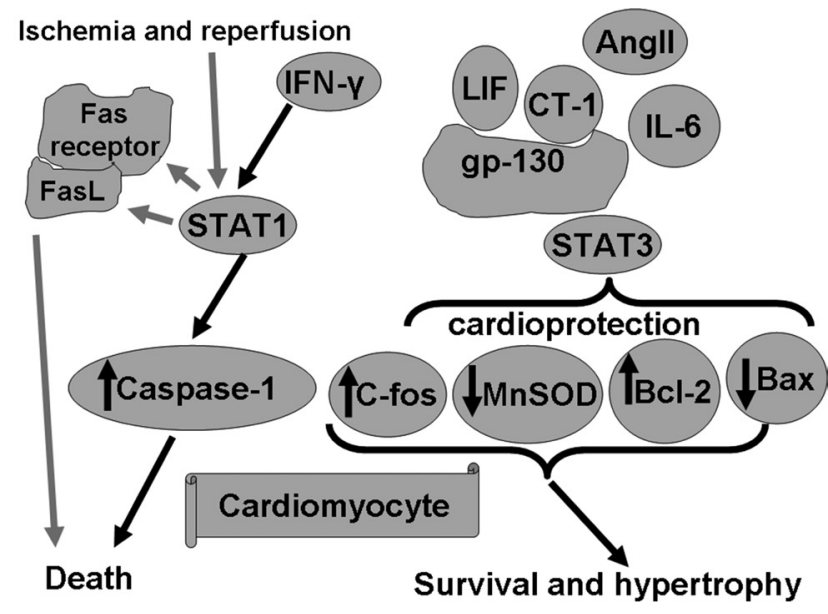

Ischemia and reperfusion, and also IFN- $\gamma$, trigger upregulation of STAT1, which interacts with the FAS receptor and FASL complex. STAT1 stimulates an increase of caspase-1 activity. Caspase-1 together with FAS receptor and FASL complex lead to cell death. LIF1 and CT-1 via gp130 and Ang II with IL- 6 induce activation of STAT3. STAT3 upregulates BCl-2 and C-fOS and downregulates MnSOD and Bax. STAT3 mediated actions contribute to hypertrophy and survival of cardiomyocytes.

\section{The therapeutic implications of STAT proteins - current state of the art and future perspectives}

The inflammatory background of cardiac remodeling should be emphasized in this review. ${ }^{25}$ Particularly, there is a magnitude of proteins that take part in events leading to heart failure, e.g. tumor necrosis factor alpha (TNF- $\alpha$ ), Fas (apoptosis stimulating fragment) protein, TRAIL (TNF- $\alpha$ related apoptosis-inducing ligand) protein, activin $\mathrm{A}, \mathrm{C}$ reactive protein, lipoprotein-associated phospholipase a2 (Lp-PLA2), myeloperoxidase and pentraxin-3, to mention the most important ones. ${ }^{25}$ Interleukin 6 (IL-6) also belongs to this group. ${ }^{25}$ IL- 6 is strongly linked to the NYHA stage of heart failure and higher levels of IL-6 ominously affect patients' survival. In the elderly population, increase of IL- 6 causes a high risk of future heart failure. ${ }^{25}$ IL-6 is the only one which mediates its effects via STAT3 action, as IL- 6 mediated acute inflammation and subsequent reactive STAT3-dependant cardiomyocyte proliferation were induced in neonatal mouse myocardium by microinjected immunogenic zymosan A to open a new horizon for cardiac regenerative medicine. ${ }^{26}$

Before some of the major clinical implications of STAT3 are presented here, it should be emphasized that STAT3 is recognized as a key but also truly pleiotropic, cardioprotective molecular factor acting as a transcriptional repressor in regulation of the ubiquitin-proteasome system, controller of cellular homeostasis of cardiomyocytes, inducer of gene expression by non-canonical pathways and mediator of nongenomic actions covering microtubule stability, mitochondrial respiration, and autophagy, with an impact on proliferation, differentiation and survival in cardiac myocytes and fibroblasts. ${ }^{27,28}$

First of all, cardiac fibroblasts, as major cardiac remodeling drivers, actively participate in myocardial remodeling in hyperglycemia and hypertension. ${ }^{29}$ Together, high levels of angiotensin II and glucose levels occur in diabetes, so it is not surprising that myocardial remodeling is so accelerated in diabetes. Hyperglycemia and Ang II increased tyrosine phosphorylation of both JAK2 and STAT3, and also enhanced collagen I deposition in cardiac fibroblasts diabetes. ${ }^{29}$ However, when atrial muscle tissue and patients' sera were investigated, it turned out that the activity of STAT3 and JAK2 was higher in nondiabetic patients (ND) than in individuals with uncontrolled diabetes (UD) in these cellular contents. ${ }^{30}$ Furthermore, the antiapoptotic proteins $\mathrm{Mcl}-1, \mathrm{Bcl}-2$ and $\mathrm{p}-\mathrm{Akt}$ were elevated, while the expressions of apoptotic proteins $\mathrm{p}-\mathrm{Bc} 12 / \mathrm{Bc} 12$ and caspase 3 were reduced in UD in comparison to ND. ${ }^{30}$ In addition, cardiopulmonary bypass $(\mathrm{CPB})$ event was associated with lower phosphorylation levels of STAT3 compared to patients with no CPB. ${ }^{30}$

STAT3 protects the myocardium and counteracts cardiac remodeling in acute myocardial cardiac event injury. ${ }^{31}$ STAT3 rapid phosphorylation follows ligation of mice coronary artery and intrinsic activation of STAT3 persists in the subacute phase of myocardial infarction. Several consequences followed the knocking of STAT3 activity by tamoxifen in rodent myocardium (tamoxifen (TM)-inducible cardiac-specific STAT3 knockout (STAT3 iCKO) mice). Namely, capillary density was lowered. Moreover, MI associated mortality was elevated. Furthermore, severe cardiac hypertrophy was induced in the border zone close to MI focus. Finally, cardiac fibrosis was favored by increased expressions of fibrosis-related gene transcripts, including matrix metalloproteinase 9 , procollagen 1 , and procollagen $3 .^{31}$

Atrial fibrillation (AF) is such an important factor of cardiovascular disease-related morbidity and mortality that Zheng et al. investigated the role of AT1, Ang II and 
STAT3 in atrial cardiomyocytes that underwent fibrillation as a consequence of ischemic hypoxia. By stimulation of its receptor AT1, Ang-II activated STAT3 via its tyrosine and serine phosphorylation in these myocytes. STAT3 affected the transcription of MMP1 and MMP2 of atrial fibroblasts. Finally, Ang II favored a potent signal for apoptosis: namely it contributed to translocation of cytochrome $\mathrm{C}$ mitochondria to the cytoplasm of atrial myocytes and this action was abolished by losartan. Thus, STAT3 is regarded as a crucial molecule on the Ang-II/AT1 receptor/ STAT3 signaling pathway, which is engaged in remodeling of the cardiac atrium. ${ }^{32}$ Interleukin-27 (IL-27), a recently discovered member of the IL- 6 family, is elevated in patients with coronary heart disease. ${ }^{33}$ IL-27 was proved to activate STAT3 by receptor subunit gp130, and this activation was reversible with application of a gp130-neutralizing antibody in rodent hearts. IL-27 and IL-6 were shown to remarkably limit cell damage after induction of severe hypoxia in $\mathrm{H} 9 \mathrm{c} 2$ cardiomyoblasts and primary rat neonatal cardiomyocytes by appreciation of lactate dehydrogenase release and crystal violet staining in experimentally induced ischemia/reperfusion (IR) injury. ${ }^{33}$

Before discussing the relationships of STAT3 with pharmacological agents, it is worth noting what STAT3 has in common with "sports". ${ }^{44}$ STAT3 plays a role in the prophylaxis and prevention of cardiac insufficiency with linkage to physical exercise. ${ }^{34}$ Namely, after physical activity (precisely, the treadmill exercise of mice), the phosphorylated (p) signal transducer and activator of transcription 3 was increased in the gastrocnemius and heart muscles, with an elevation of serum IL-6 and IL-6 receptor (IL-6R), and IL-6R of gastrocnemius and heart muscle origin. Such remarkable findings could signify that keeping our skeletal muscles in good shape helps our myocardium in its cardioprotective aspect via elevation of circulating IL- $6 .{ }^{34}$ Somewhat in line with the findings of McGinnis et al., it was revealed that if ischemia/reperfusion events are triggered in a remote organ, the myocardium can acquire a higher resistance to ischemic injury as a result. ${ }^{34,35}$ Under remote ischemic preconditioning (RIPC), there was decrease of serum troponin I levels with an accompanying increase of STAT5 phosphorylation in patients undergoing coronary artery bypass surgery in comparison with control patients without RIPC. ${ }^{35}$ In a pig experimental model of remote ischemic preconditioning, it was proved that RIPC limited infarct size and augmented STAT3 phosphorylation while STAT5 remained decreased in the early phase of reperfusion in both the RIPC and control groups. Such cardioprotection can be transferable with plasma from pigs to isolated rat hearts. Moreover, it was concluded that the cardioprotective effect of STAT3 signaling and function is the same in RIPC and ischemic postconditioning. ${ }^{36}$

There are plenty of pharmacological ways to regulate STAT3 activity, so our task was to focus only on those examples which are most essential for internal medicine practitioners. If mocetinostat (a selective HDAC inhibi- tor tested in oncologic disorders) was injected intraperitoneally, pSTAT3 and histone deacetylases (HDAC1 and HDAC2), pro-fibrotic markers, collagen-1, fibronectin Connective Tissue Growth Factor (CTGF) and IL-6 levels were decreased in cardiomyocytes, while cardiac fibroblasts expressed less of collagen I and III, fibronectin and TIMP-1 (tissue inhibitors of metalloproteinas-1) in rats which developed congestive heart failure CHF as a consequence of MI (myocardial infarction) due to coronary artery occlusion. ${ }^{37}$ With a decrease of levels of these factors, fibrotic scar total collagen quantity was limited. Thus, it can be concluded that the anti-fibrotic properties of mocetinostat are mediated by a decrease of IL-6/STAT3 signaling in CHF. ${ }^{37}$

Wang L. et al. studied the impact of losartan (a selective angiotensin II type 1 (AT1) receptor antagonist) on myocardial interstitial fibrosis in male Wister rats with streptozotocin (STZ)-induced diabetes and the accompanying cardiomyopathy (DCM). ${ }^{38}$ In opposition to rats with DCM but without losartan treatment, the myocardial levels of p-JAK2 and p-STAT3 decreased in DCM rodent hearts as a result of losartan treatment, with a decline of TGF- $\beta 1$ cardiac collagen quantity and LVEDP (left ventricular end-diastolic pressure) with augmentation of LVSP (left ventricular systolic pressure) $\pm \mathrm{dp} / \mathrm{dt}^{38}$

Lovastatin transiently increased the level of phosphorylated STAT3 as well as protein phosphatase 1 until miR-21 microRNA levels were significantly diminished. ${ }^{39}$ On the other hand, simvastatin decreased expression of pSTAT3 in cardiomyocytes, which was previously elevated in hypertrophy of the heart. Simvastatin was shown to counteract that kind of cardiac hypertrophy, which can be induced by isoproterenol in rats. ${ }^{40}$

\section{Fig. 2. Current therapeutic implications of STAT3 on cardiomyocytes}

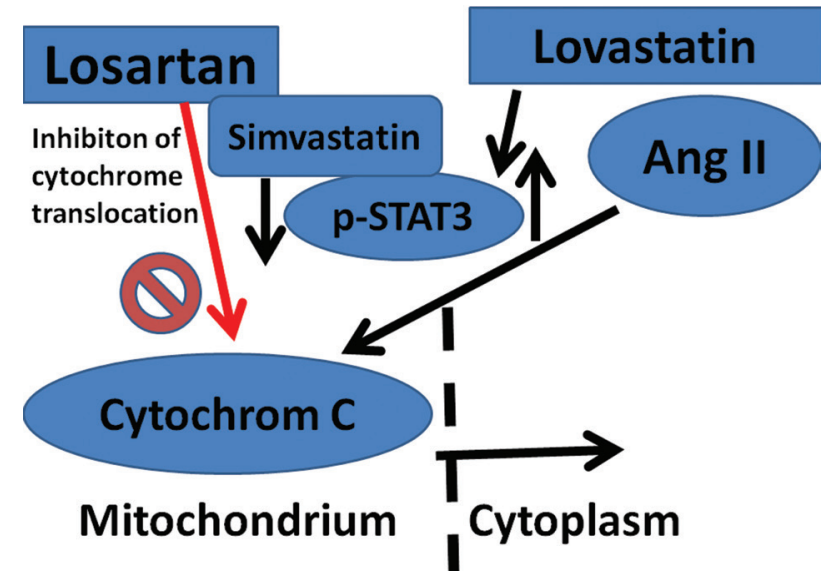

Ang II and lovastatin were both shown to increase the phosphorylated level of STAT3 in myocardial cells. However, only Ang II was shown to increase cardiomyocyte apoptosis via stimulation of the translocation of cytochrome $\mathrm{C}$ from mitochondrium to cytoplasm. In contrast, losartan inhibits the translocation of cytochrome $C$ from mitochondrium to cytoplasm. Thus, losartan exerts anti-apoptotic properties on cardiac myocytes. Simvastatin limited expression of PSTAT3 in cardiomyocytes which was previously elevated in isoproterenol (ISO)-evoked hypertrophy of the rodent heart. ${ }^{40}$ Simvastatin was shown to counteract that kind of cardiac hypertrophy. 


\section{Conclusion}

STAT proteins are engaged in the differentiation and survival of cellular components not only in the vascular bed but also in the cardiac walls. ${ }^{41}$ Induction of STAT proteins was traditionally associated with inflammatory response. ${ }^{25,42}$ Their overexpression can affect the regulation of ischemic cardiac damage during hypoxia. So in the future, STAT proteins could serve as targets for different sorts of treatment that would slow down the progress of coronary atherosclerosis and reduce hypoxic injury of the heart. To sum up, pathways of intracellular signaling can be elucidated thanks to STAT proteins in the heart. Particularly STAT3 is emerging as the most essential representative of this protein family for both classical pharmacological counteractions of myocardial remodeling with such drugs as losartan or lovastatin and for such novel experimental modes of treatment as microRNAs.

\section{References}

1. Akira S. Functional roles of STAT family proteins: Lessons from knockout mice. Stem Cells. 1999;17:138-146.

2. Wang Y, Malabarba MG, Nagy ZS, Kirken RA. Interleukin 4 regulates phosphorylation of serine 756 in the transactivation domain of Stat6. Roles for multiple phosphorylation sites and Stat6 function. J Biol Chem. 2004;279:25196-25203.

3. Calo V, Migliavacca M, Bazan V, et al. STAT proteins: From normal control of cellular events to tumorigenesis. J Cell Physiol. 2003;197:157-168.

4. Varinou L, Ramsauer K, Karaghiosoff M, et al. Phosphorylation of the Stat1 transactivation domain is required for fullfledged IFN-gamma-dependent innate immunity. Immunity. 2003;19:793-802.

5. Bromberg J. Stat proteins and oncogenesis. J Clin Invest. 2002:109:1139-1142.

6. Kunisada K, Tone E, Fujio Y, Matsui H, Yamauchi-Takihara K, Kishimoto T. Activation of gp130 transduces hypertrophic signals via STAT3 in cardiac myocytes. Circulation. 1998;98:346-352.

7. Funamoto $M$, Fujio $Y$, Kunisada K, et al. Signal transducer and activator of transcription 3 is required for glycoprotein 130-mediated induction of vascular endothelial growth factor in cardiac myocytes. J Biol Chem. 2000;275:10561-10566.

8. Osugi T, Oshima Y, Fujio Y, et al. Cardiac-specific activation of signal transducer and activator of transcription 3 promotes vascular formation in the heart. J Biol Chem. 2002;277:6676-6681.

9. Hilfiker-Kleiner D, Hilfiker A, Fuchs M, et al. Signal transducer and activator of transcription 3 is required for myocardial capillary growth, control of interstitial matrix deposition, and heart protection from ischemic injury. Circ Res. 2004;95:187-195.

10. Negoro $S$, Kunisada $K$, Fujio $Y$, et al. Activation of signal transducer and activator of transcription 3 protects cardiomyocytes from hypoxia/reoxygenation-induced oxidative stress through the upregulation of manganese superoxide dismutase. Circulation. 2001:104:979-981.

11. Wollert KC, Taga T, Saito M, et al. Cardiotrophin-1 activates a distinct form of cardiac muscle cell hypertrophy. Assembly of sarcomeric units in series VIA gp130/leukemia inhibitory factor receptordependent pathways. J Biol Chem. 1996;271:9535-9545.

12. Kunisada K, Negoro S, Tone E, et al. Signal transducer and activator of transcription 3 in the heart transduces not only a hypertrophic signal but a protective signal against doxorubicin-induced cardiomyopathy. Proc Natl Acad Sci USA. 2000;97:315-319.

13. Podewski EK, Hilfiker-Kleiner D, Hilfiker A, et al. Alterations in Janus kinase (JAK)-signal transducers and activators of transcription (STAT) signaling in patients with end-stage dilated cardiomyopathy. Circulation. 2003;107:798-802.
14. Jacoby JJ, Kalinowski A, Liu MG, et al. Cardiomyocyte-restricted knockout of STAT3 results in higher sensitivity to inflammation, cardiac fibrosis, and heart failure with advanced age. Proc Nat/ Acad Sci USA. 2003;100:12929-12934.

15. Sano M, Fukuda K, Kodama $H$, et al. Autocrine/Paracrine secretion of IL- 6 family cytokines causes angiotensin II-induced delayed STAT3 activation. Biochem Biophys Res Commun. 2000;269:798-802.

16. Kodama H, Fukuda K, Pan J, et al. Biphasic activation of the JAK/ STAT pathway by angiotensin II in rat cardiomyocytes. Circ Res. 1998;82:244-250.

17. Booz GW, Day JN, Speth R, Baker KM. Cytokine G-protein signaling crosstalk in cardiomyocytes: Attenuation of Jak-STAT activation by endothelin-1. Mol Cell Biochem. 2002;240:39-46.

18. Hattori R, Maulik N, Otani H, et al. Role of STAT3 in ischemic preconditioning. J Mol Cell Cardiol. 2001;33:1929-1936.

19. Stephanou A, Brar BK, Scarabelli TM, et al. Ischemia-induced STAT-1 expression and activation play a critical role in cardiomyocyte apoptosis. J Biol Chem. 2000;275:10002-10008.

20. Stephanou A, Scarabelli TM, Brar BK, et al. Induction of apoptosis and Fas receptor/Fas ligand expression by ischemia/reperfusion in cardiac myocytes requires serine 727 of the STAT- 1 transcription factor but not tyrosine 701. J Biol Chem. 2001;276:28340-28347.

21. Fujio Y, Kunisada K, Hirota H, Yamauchi-Takihara K, Kishimoto T. Signals through gp130 upregulate bcl-x gene expression via STAT1-binding cis-element in cardiac myocytes. J Clin Invest. 1997;99:2898-2905.

22. Kawai M, Kawashima S, Sakoda T, et al. Ral GDP dissociation stimulator and Ral GTPase are involved in myocardial hypertrophy. Hypertension. 2003;41:956-962.

23. Sheng Z, Knowlton K, Chen J, Hoshijima M, Brown JH, Chien KR. Cardiotrophin 1 (CT-1) inhibition of cardiac myocyte apoptosis via a mitogen-activated protein kinase-dependent pathway. Divergence from downstream CT-1 signals for myocardial cell hypertrophy. J Biol Chem. 1997;272:5783-5791.

24. Yamaura G, Turoczi T, Yamamoto F, Siddqui MA, Maulik N, Das DK. STAT signaling in ischemic heart: A role of STAT5A in ischemic preconditioning. Am J Physiol Heart Circ Physiol. 2003;285:H476-482.

25. Oikonomou E1, Tousoulis D, Siasos G, Zaromitidou M, Papavassiliou AG, Stefanadis C. The role of inflammation in heart failure: New therapeutic approaches. Hellenic J Cardiol. 2011;52:30-40.

26. Han C, Nie Y, Lian H, Liu R, He F, Huang H, Hu S. Acute inflammation stimulates a regenerative response in the neonatal mouse heart. Cell Res. 2015 Sep 11. doi: 10.1038/cr.2015.110. [Epub ahead of print]

27. Zouein FA, Kurdi M, Booz GW. Dancing rhinos in stilettos: The amazing saga of the genomic and nongenomic actions of STAT3 in the heart. JAKSTAT. 2013; 2:e24352.

28. Haghikia A, Ricke-Hoch M, Stapel B, Gorst I, Hilfiker-Kleiner D. STAT3, a key regulator of cell-to-cell communication in the heart. Cardiovasc Res. 2014;102:281-289

29. Fiaschi T, Magherini F, Gamberi T, et al. Hyperglycemia and angiotensin II cooperate to enhance collagen I deposition by cardiac fibroblasts through a ROS-STAT3-dependent mechanism. Biochim Biophys Acta. 2014;1843:2603-2610.

30. Owais K, Huang T, Mahmood F, et al. Cardiopulmonary bypass decreases activation of the Signal Transducer and Activator of Transcription 3 (STAT3) pathway in diabetic human myocardium. Ann Thorac Surg. 2015 pii: S0003-4975(15)00835-8. [Epub ahead of print].

31. Enomoto D, Obana M, Miyawaki A, Maeda M, Nakayama H, Fujio Y. Cardiac-specific ablation of the STAT3 gene in the subacute phase of myocardial infarction exacerbated cardiac remodeling. Am J Physiol Heart Circ Physiol. 2015;309:H471-480.

32. Zheng LY, Zhang MH, Xue JH, Li Y, Nan Y, Li MJ, Wang J, Du XP. Effect of angiotensin II on STAT3 mediated atrial structural remodeling. Eur Rev Med Pharmacol Sci. 2014;18:2365-2377.

33. Ma MC, Wang BW, Yeh TP, et al. Interleukin-27, a novel cytokine induced by ischemia-reperfusion injury in rat hearts, mediates cardioprotective effects via the gp130/STAT3 pathway. Basic Res Cardiol. 2015;110:22.

34. McGinnis GR, Ballmann C, Peters B, et al. Interleukin-6 mediates exercise preconditioning against myocardial ischemia reperfusion injury. Am J Physiol Heart Circ Physiol. 2015;308:H1423-1433. 
35. Heusch G, Musiolik J, Kottenberg E, Peters J, Jakob H, Thielmann M. STAT5 activation and cardioprotection by remote ischemic preconditioning in humans: Short communication. Circ Res. 2012;110: 111-115.

36. Skyschally A, Gent S, Amanakis G, Schulte C, Kleinbongard P, Heusch G Across-species transfer of protection by remote ischemic preconditioning with species-specific myocardial signal transduction by reperfusion injury salvage kinase and survival activating factor enhancement pathways. Circ Res. 2015;117:279-288.

37. Nural-Guvener H, Zakharova L, Feehery L, Sljukic S, Gaballa M. Antifibrotic effects of class I HDAC inhibitor, mocetinostat is associated with IL-6/Stat3 signaling in ischemic heart failure. Int J Mol Sci. 2015;16:11482-11499.

38. Wang L, Li J, Li D. Losartan reduces myocardial interstitial fibrosis in diabetic cardiomyopathy rats by inhibiting JAK/STAT signaling pathway. Int J Clin Exp Pathol. 2015;8:466-473.

39. Guo W, Liu H, Li L, Yang M, Du A. Regulation of lovastatin on a key inflammation-related microRNA in myocardial cells. Chin Med $J$ (Engl). 2014;127:2977-2981.

40. Al-Rasheed NM, Al-Oteibi MM, Al-Manee RZ, et al. Simvastatin prevents isoproterenol-induced cardiac hypertrophy through modulation of the JAK/STAT pathway. Drug Des Devel Ther. 2015;9:3217-3229.

41. Wincewicz A, Sulkowska M, Rutkowski R, et al. STAT1 and STAT3 as intracellular regulators of vascular remodeling. Eur J Intern Med. 2007;18:267-271.

42. Wincewicz A, Moniuszko T, Sulkowska M, Rutkowski R, Koda M, Sulkowski S. Involvement of STAT proteins in the pathogenesis of autoimmune diseases. Adv Clin Exp Med. 2005;14:785-790. 